\title{
THE MODULAR REPRESENTATION ALGEBRA OF GROUPS WITH SYLOW 2-SUBGROUP $z_{2} \times z_{2}$
}

\author{
S. B. CONLON
}

(Received 18 January 1965)

Let $k$ be a field of characteristic 2 and let $G$ be a finite group. Let $A(G)$ be the modular representation algebra ${ }^{1}$ over the complex numbers $C$, formed from $k G$-modules ${ }^{2}$. If the Sylow 2-subgroup of $G$ is isomorphic to $Z_{2} \times Z_{2}$, we show that $A(G)$ is semisimple ${ }^{1}$. We make use of the theorems proved by Green [4], and the results of the author concerning $A\left(\AA_{4}\right)[2]$, where $\mathscr{A}_{4}$ is the alternating group on 4 symbols.

\section{Generalities on representation algebras}

Let $A$ be any commutative linear algebra over the complex number field $C$. A point of $A$ is a non-zero algebra homomorphism

$$
\phi: A \rightarrow C \text {. }
$$

Thus $\phi(A)=C . A$ is said to be semisimple if for each non-zero element $a \in A$, there exists a point $\phi$ of $A$ such that $\phi(a) \neq 0$. If $\operatorname{dim}_{C} A=r$ is finite, $A$ is semisimple if and only if $A$ has $r$ points; $A$ is then the direct sum of $r$ copies of $C$.

Proposition 1. If $B$ is an ideal of $A$ such that both $A / B$ and $B$ are semisimple, then $A$ is semisimple.

Proof. Take $a \in B$, and let $\phi$ be a point of $B$ such that $\phi(a) \neq 0$. We extend ${ }^{3} \phi$ to be a point of $A$ by noting that, as $\phi(B)=C$, there exists in $B$ an element $b$ such that $\phi(b)=1$. For $x$ any element of $A$, we define $\phi(x)=\phi(x b)$.

Secondly let $a \notin B$. Thus there exists a point $\phi$ of $A / B$ such that $\phi(a+B) \neq 0$. But $\phi$ can be regarded as a point of $A$ which is zero on $B$. Thus $\phi(a) \neq 0$ and so $A$ is semisimple.

Let $k$ be an arbitrary field and $G$ a finite group. Let $M$ be a $k G$-module

1 We adopt the definitions and notation of Green in [4].

- A $k G$-module is a finitely generated $k$-module on which $G$ acts as a group of left operators. $k G$ is the group algebra on $G$ over $k$.

- As in lemma 6 of [4]. 
(of finite $k$-dimension), and write $\{M\}$ for the class of modules $k G$-isomorphic to $M$ (or simply the "class of $M$ "). As in [4] we form the modular representation algebra $A(G)$ as an algebra over the complex numbers $C$ in which sum corresponds to direct sum of modules and multiplication to tensor product of $k G$-modules. A basis for $A(G)$ over $C$ is provided by the indecomposable $k G$-module classes. $k_{G}$ will denote the trivial $k G$-module, and $1_{G}=\left\{k_{G}\right\}$ its class. Then $A(G)$ is a commutative algebra over $C$ with identity $1_{G}$.

Let $\theta: H \rightarrow G$ be a homomorphism of groups, $L$ a $k H$-module and $M$ a $k G$-module. Then $\theta^{*} M$ will denote the restricted $k H$-module, where the operation of a group element $h \in H$ on $m \in M$ is given by

$$
h \cdot m=\theta(h) m \text {. }
$$

$\theta_{*} L$ will denote the induced $k G$-module

$$
k G \otimes_{k H} L,
$$

where $k G$ is regarded as a right $k H$-module by means of $\theta$. Thus we get induced linear maps:

$$
\theta^{*}: A(G) \rightarrow A(H), \quad \theta_{*}: A(H) \rightarrow A(G) .
$$

$\theta^{*}$ is an algebra homomorphism, while for $\theta_{*}$ we have the identity

$$
\theta_{*} L \otimes M \approx \theta_{*}\left(L \otimes \theta^{*} M\right) \text {. }
$$

Here ' $\otimes$ ' denotes Tensor (or Kronecker) product of the representation modules. In particular, if $H$ is a subgroup of $G$, with $\theta$ the embedding map, we write $M_{H}=\theta^{*} M$, and $L^{\sigma}=\theta_{*} L$; also $\theta^{*}, \theta_{*}$ coincide with the maps $\gamma_{G H}, t_{H G}$ respectively of Green [4].

If $H$ is a normal subgroup of $G$, and $L$ is $k H$-module, let $S$ denote the set of elements $s \in G$ such that $s \otimes_{k H} L \approx L$ as left $k H$-modules. Then $S$ is a subgroup of $G$ containing $H$ and is called the stabilizer of $L$ in $G$. If $S=G$, we say that $L$ is stable in $G$. $\S 2$ of [1] contains the following theorem: (2) If $L$ is indecomposable, then $L^{G}$ decomposes according to the decomposition of a certain twisted group algebra on $S / H$ into one-sided indecomposable ideals.

$\left(2^{\prime}\right)$ It should be noted that twisted group algebras on cyclic groups are always isomorphic to the group algebras.

Proposition 2. If $G_{1} G_{2}$ is the direct product of finite groups $G_{1}, G_{2}$ and if $\left(\left|G_{1}\right|, p\right)=1$, where $p$ is the characteristic of $k$, or if $k$ has characteristic 0 , then

- For proof of (1), see p. 268 of [3]. 


$$
A\left(G_{1} G_{2}\right) \approx A\left(G_{1}\right) \otimes_{\mathrm{c}} A\left(G_{2}\right) .
$$

Proof. Write $G=G_{1} G_{2}$, and let $\sigma_{i}: G \rightarrow G_{i}$ be the natural homomorphisms $(i=1,2)$. Then we have .

$$
\sigma_{i}^{*}: A\left(G_{i}\right) \rightarrow A(G),
$$

and combining these we get an algebra homomorphism

$$
\sigma^{*}=\sigma_{1}^{*} \otimes \sigma_{2}^{*}: A\left(G_{1}\right) \otimes_{C} A\left(G_{2}\right) \rightarrow A(G),
$$

which we show to be an isomorphism.

By Higman's theorem 1 in [5], every indecomposable $k G$-module can be considered as a direct summand of $L^{G}$, where $L$ is an indecomposable $k G_{2}$-module. Now $L$ is stable in $G$ and the twisted group algebra of (2) is the group algebra $k G_{1}$. Indeed the endomorphisms $\theta_{\alpha, \beta}$ of $L$ in the analysis of $\S 2$ of [1] may all be taken to be the identity automorphism, and for $g \in G$ we may take

$$
D_{0}=\lambda\left(\sigma_{2}(g)\right) \quad \text { (Notation as in } \S 2 \text { of [1]), }
$$

where $\lambda$ is a $G_{2}$-representation afforded by $L$. If $\pi$ is a principal indecomposable $G_{1}$-representation, the typical indecomposable $G$-representation $\psi$ has the form

$$
\psi(g)=\pi\left(\sigma_{1}(g)\right) \otimes \lambda\left(\sigma_{2}(g)\right),
$$

analogously to proposition 1 of $\S 2$ in [1]. Hence the indecomposable $k G$ modules have the form ${ }^{5}$

$$
P \not L \text {, }
$$

where $P$ and $L$ are indecomposable $k G_{1^{-}}$and $k G_{2^{-}}$-modules respectively. Then $\sigma^{*}\left\{P \otimes_{C} L\right\}=\{P \# L\}$, and $\sigma^{*}$ is onto.

Moreover, if $P, L$ are indecomposable, and

$$
P \not L \approx P^{\prime} \not L^{\prime} \quad \text { (as } k G \text {-modules), }
$$

by restricting to $G_{1}$ and $G_{2}$ it follows that $P \approx P^{\prime}, L \approx L^{\prime}$. As $\left\{P \otimes_{C} L\right\}$, $\{P \# L\}$ form free bases over $C$ for $A\left(G_{1}\right) \otimes_{C} A\left(G_{2}\right), A\left(G_{1} G_{2}\right)$ respectively, $\sigma^{*}$ is $1-1$ and so $\sigma^{*}$ is an isomorphism. stable.

Identity (1) has the following consequence when representations are

Proposition 3. Let $H$ be a normal subgroup of $G$ and suppose all the indecomposable $k H$-modules are stable in $G$. Then $A(H)$ isomorphic to an ideal direct summand of $A(G)$.

Proof. Let $\phi: H \rightarrow G$ be the inclusion homomorphism and let $L$ be a $k H$-module. Define

5 This is the outer tensor product as defined on p. 315 of [3]. 


$$
\sigma\{L\}=\frac{1}{m} \phi_{*}\{L\} \quad(m=G: H)
$$

and then $\sigma$ induces a homomorphism of $A(H)$ into $A(G)$. For if $L, L^{\prime}$ are $k H$-modules, we have

$$
\begin{aligned}
\sigma\left(\{L\} \cdot\left\{L^{\prime}\right\}\right) & =\sigma\left\{L \otimes L^{\prime}\right\} \\
& =\frac{1}{m} \phi_{*}\left\{L \otimes L^{\prime}\right\} \\
& =\frac{1}{m^{2}} \phi_{*}\left\{L \otimes \phi^{*}\left(\phi_{*}\left(L^{\prime}\right)\right)\right\} \quad \text { (as } L^{\prime} \text { is stable) } \\
& \left.=\frac{1}{m^{2}}\left\{\phi_{*}(L) \otimes \phi_{*}\left(L^{\prime}\right)\right\} \quad \text { (by }(1)\right)
\end{aligned}
$$

i.e. $\quad \sigma\left(\{L\} \cdot\left\{L^{\prime}\right\}\right)=\sigma\{L\} \cdot \sigma\left\{L^{\prime}\right\}$.

Now $I=\sigma\left(\mathbf{1}_{H}\right)$ is an idempotent of $A(G)$ from (5), and if $M$ is any $k G$-module it follows from (1) that

Again from (5)

$$
I \cdot\{M\}=\sigma\left\{\phi^{*} M\right\} \text {. }
$$

$$
I \cdot \sigma\{L\}=\sigma\{L\}
$$

and so the image of $\sigma$ is the ideal direct summand $I \cdot A(G)$ of $A(G)$.

Furthermore the restriction $\rho$ of $\phi^{*}$ to $I \cdot A(G)$ satisfies the conditions,

and

$$
\rho \sigma=\text { identity homomorphism on } A(H) \text {, }
$$

$$
\sigma \rho=\text { identity homomorphism on } I \cdot A(G),
$$

and so $\sigma$ is an isomorphism of $A(H)$ onto $I \cdot A(G)$.

Thus we see that in $A\left(G_{1}\right) \otimes_{C} A\left(G_{2}\right)\left(\approx A\left(G_{1} G_{2}\right)\right)$ of proposition 2 we have direct summands isomorphic to $A\left(G_{1}\right)$ and $A\left(G_{2}\right)$.

We will require the following

PROPOSITION 4. If $H$ is a normal subgroup of $G$, then the decomposition of $A(G / H)$ as a direct sum of ideals gives rise to a corresponding one for $A(G)$.

Proof. Consider the natural map $\theta: G \rightarrow G / H$. This induces a monomorphism $\theta^{*}: A(G / H) \rightarrow A(G)$. Moreover $\theta^{*}\left(1_{G / H}\right)=1_{G}$. Thus any decomposition of the identity $\mathbf{1}_{G / H}$ of $A(G / H)$ into the sum of idempotents is carried over by $\theta^{*}$ into $A(G)$, and similarly for the ideals generated by these idempotents in their respective algebras.

Let $P$ be a subgroup of $G$, and write $A_{P}(G)$ for the $C$-subspace of $A(G)$ spanned by the symbols $\{L\}$ for all $P$-projective $k G$-modules $L$. Write 
$A_{P}^{\prime}(G)$ for the subspace of $A(G)$ spanned by the symbols $\{L\}$ for all $H$ projective $k G$-modules $L$, where $H \leqq G P, H \neq_{G} P^{5^{\prime}}$. As in [4], $A_{P}(G)$ and $A_{P}^{\prime}(G)$ are ideals of $A(G)$, with $A_{P}^{\prime}(G) \subseteq A_{P}(G)$.

Write $W_{P}(G)=A_{P}(G) / A_{P}^{\prime}(G)$.

(6) If $k$ has characteristic $p$, then Green in [4] shows that $A(G)$ is semisimple if, for each $p$-subgroup $P$ of $G, W_{P}(N(P))$ is semisimple, where $N(P)$ is the normalizer of $P$ in $G$.

(7) Proposition 3 in [2] shows that if we take the trivial $p$-subgroup $P=\{e\}$, then $W_{P}(N(P))=A_{P}(G)$ is the "projective ideal" of $A(G)$, which is an ideal direct summand of $A(G)$ consisting of the direct sum of a finite number of copies of $C$. Hence for $P=\{e\}, W_{P}(N(P))$ is semisimple. We denote the projective ideal of $A(G)$ by $A_{0}(G)$.

Finally it should be noted that as far as the question of the semisimplicity of $A(G)$ is concerned we can assume $k$ to be algebraically closed. For if not let $k^{*}$ be its algebraic closure and let $A^{*}(G)$ be the corresponding modular representation algebra. Proposition 1 and (3) of [2] show there is a natural monomorphism

$$
A(G) \rightarrow A^{*}(G),
$$

and so, if $A^{*}(G)$ is semisimple, the restrictions of its points to $A(G)$ ensure the semisimplicity of $A(G)$.

\section{Representation algebras of $\mathscr{V}_{4}$ and $\mathscr{A}_{4}$}

Let $k$ be an algebraically closed field of characteristic 2, let $\mathscr{V}_{4}=Z_{2} \times Z_{2}$, be the Klein 4-group and let $\mathscr{A}_{4}$ be the alternating group on 4 symbols. We shall consider $\mathscr{V}_{4}$ to be identified with the Sylow 2-subgroup of $\mathscr{A}_{4}$. The following facts are proved in [2].

The indecomposable $k \mathscr{V}$-module classes may be written

$$
A_{0}=B_{0}, A_{n}, B_{n}, C_{n}(\pi), D,
$$

where $n>0$, and $\pi \in k \cup\{\infty\}$. If we write

$$
\mathscr{r}_{4}=\left\{x, y \mid x^{2}=y^{2}=e, x y=y x\right\},
$$

then the vertices of these classes are as follows:

(9i) $A_{0}, A_{n}(n>0), B_{n}(n>0), C_{n}(\pi)(n>1)$, and $C_{1}(\pi)(\pi \neq 0,1, \infty)$ have vertex $\mathscr{V}_{4}$,

(9ii) $C_{1}(0), C_{1}(1), C_{1}(\infty)$ have vertices $\{y\},\{x y\},\{x\}$ respectively (order 2),

(9iii) and $D$ has vertex $\{e\}$ (order 1).

$D$ is the regular (indecomposable) module class.

b' $H \leqq_{c} P$ means that there exists an element $x \in G$ such that $x^{-1} H x \leqq P$, etc. 
We require the following products:

(10i) $B_{m} C_{n}(\pi)=A_{m} C_{n}(\pi)=C_{n}(\pi) \bmod A_{0}\left(\mathscr{V}_{4}\right)(n>0, m \geqq 0)$, (10ii) $C_{m}(\pi) C_{n}\left(\pi^{\prime}\right)=0$, if $\pi \neq \pi^{\prime}$, $2 C_{n}(\pi)$, if $\pi=\pi^{\prime}, m \geqq n$, except that $C_{1}(\pi) C_{1}(\pi)=C_{2}(\pi)$ if $\pi \neq 0,1$ or $\infty$, $\bmod A_{6}\left(\mathscr{V}_{4}\right)$

The representation algebra $A\left(\mathscr{V}_{4}\right)$ may be written:

$$
A\left(\mathscr{V}_{4}\right)=\left(C\left[X, \frac{1}{X}\right]+\left\{\underset{\substack{\pi, n \\ n>0}}{\oplus} C I_{n, \pi}\right\}\right) \oplus C I_{D},
$$

where $X^{m} I_{n, \pi}=I_{n, n}$ (all integers $m$ ) and where $\left\{\oplus_{n, n>0} C I_{n, n}\right\}$ is the direct sum of ideals isomorphic to $C . C I_{D}$ is the projective ideal $A_{e}\left(\mathscr{V}_{t}\right)$. Here we have the following identifications module $A_{8}\left(\mathscr{V}_{4}\right)$ :

$$
\left.\begin{array}{llrl}
X^{n} & =A_{n} & & (n \geqq 0), \\
X^{-n} & =B_{n} & & (n \geqq 0), \\
I_{1, \pi} & =\frac{1}{2} C_{1}(\pi), & & \\
I_{n, \pi} & =\frac{1}{2}\left(C_{n}(\pi)-C_{n-1}(\pi)\right) & & (n>1),
\end{array}\right\} \text { when } \pi=0,1 \text { or } \infty,
$$

$A\left(\mathscr{V}_{4}\right)$ is semisimple. We may write

$$
W_{r_{4}}\left(\mathscr{V}_{4}\right)=C\left[X, \frac{1}{X}\right]+\left(\left\{\underset{\substack{n \neq(0,1, \infty) \\ n>0}}{\oplus} C I_{n, \pi}\right\} \oplus\left\{\underset{\substack{\pi=(0,1, \infty) \\ n>1}}{\oplus} C I_{n, \pi}\right\}\right)
$$

and as in the proof of the semisimplicity of $A\left(\mathscr{V}_{4}\right)$ in $\S 4$ of $[2], W_{\mathscr{V}_{4}}\left(\mathscr{V}_{4}\right)$ is semisimple.

$\mathscr{V}_{4} \triangleleft \mathscr{A}_{4}$ and so we can consider the stability of $k \mathscr{V}_{4}$-module classes in $\mathscr{A}_{\mathbf{4}}$. We have that

(14i) $A_{0}, A_{n}, B_{n}, C_{n}(\omega), C_{n}\left(\omega^{2}\right), D$ are stable in $\mathscr{A}_{4}$, (14ii) $C_{n}(\pi)\left(\pi \neq \omega, \omega^{2}\right)$ are not stable in $\mathscr{A}_{4}$, where $\omega$ is a primitive cube root of unity in $k$.

Say $w$ is an element of order 3 of $\mathscr{A}_{4}$ with $w^{-2} x w^{2}=w^{-1} y w=x y$. Then we have that the $k \mathscr{V}$-module class

$$
w \otimes_{k r_{0}} C_{n}(\pi)=C_{n}(\theta(\pi)) \text {. }
$$


where $\theta(\pi)=(1+\pi) / \pi$, with the obvious interpretation when $\pi=0$ or $\infty$. $\theta$ gives a permutation on $k \cup\{\infty\}$. We denote the typical class of transitivity by $\mu=\left(\pi, \theta(\pi), \theta^{2}(\pi)\right)$, but $(\omega)$ and $\left(\omega^{2}\right)$ form transitivity classes by themselves. Applying (2) together with Higman's theorem 1 in [5], we see that the indecomposable $k \mathscr{A}$-module classes can be written (see [2])

$$
\begin{aligned}
& A_{0}^{\alpha}, A_{n}^{\alpha}, B_{n}^{\alpha}, C_{n}^{\alpha}(\omega), C_{n}^{\alpha}\left(\omega^{2}\right), D^{\alpha} \\
& C_{n}^{*}(\mu),
\end{aligned}
$$

where $n>0$ and $\alpha=0,1,2$. Superscripts $\alpha$ will always be taken modulo 3 $(0,1$ or 2$)$. Note that

$$
\left(C_{n}^{*}(\mu)\right) \mathscr{V}_{4}=C_{n}(\pi)+C_{n}(\theta(\pi))+C_{n}\left(\theta^{2}(\pi)\right) \text {, and }\left(L^{a}\right)_{r_{4}}=L,
$$

where $L^{\alpha}$ is any one of $(16 i)$. The vertices of the above $k d$-module classes remain the same as the corresponding $k \mathscr{V}$, -module classes. The representation algebra $A\left(\mathscr{A}_{4}\right)$ may be written

$$
\begin{aligned}
& A\left(\mathscr{A}_{1}\right)=\left(C\left[Y_{0}, \frac{1}{Y_{0}}\right]+\left\{\underset{\substack{n>0 \\
\phi=\infty, \omega^{2}, \mu}}{\bigoplus} C I_{n 0}(\phi)\right\}\right) \\
& \oplus\left(\underset{\beta=1,2}{\oplus}\left[C\left[Y_{\beta}, \frac{1}{Y_{A}}\right]+\left\{\underset{\substack{n>0 \\
\phi=\omega, \omega^{2}}}{\oplus} C I_{n \phi}(\phi)\right\}\right]\right) \\
& \oplus(C \oplus C \oplus C) \text {, }
\end{aligned}
$$

where the last term is the projective ideal $A_{0}\left(\mathscr{A}_{4}\right)$,

$$
\begin{aligned}
& Y_{\beta}^{m} I_{n \beta}\left(\omega^{\alpha}\right)=u^{-\alpha \beta m} I_{n \beta}\left(\omega^{\alpha}\right) \quad(\beta=0,1,2 ; \alpha=1,2), \\
& Y_{0}^{m} I_{n 0}(\mu)=I_{n 0}(\mu),
\end{aligned}
$$

with $\boldsymbol{u}$ a primitive cube root of unity in $C$. We have the following identifications modulo $A_{0}\left(\mathscr{A}_{4}\right)$ :

$$
\begin{aligned}
& Y_{\beta}^{n}=\frac{1}{3}\left(A_{n}^{0}+u^{\beta} A_{n}^{1}+u^{2 \beta} A_{n}^{2}\right), \\
& Y_{\beta}^{-n}=\frac{1}{3}\left(B_{n}^{0}+u^{\beta} B_{n}^{1}+u^{2 \beta} B_{n}^{2}\right),
\end{aligned}
$$

$I_{n \beta}(\phi)=$ finite linear combination of $C_{m}^{\alpha}(\phi)$, for $\alpha, \beta=0,1,2 . A\left(\mathscr{A}_{4}\right)$ is again semisimple.

\section{3. $A(G)$ for $G$ with Sylow 2-subgroup $Z_{2} \times Z_{2}$}

Let $k$ be an algebraically closed field of characteristic 2 and $G$ a finite group with Sylow 2-subgroup isomorphic to $\mathscr{V}_{4}=Z_{2} \times Z_{2}$. To see that $A(G)$ is semisimple we use Green's theorem (6) and show that $W_{P}(N(P))$ is semisimple, where $P$ is a 2-subgroup of $G$ of order 1,2 or 4 . 
The case when $|P|=1$ has been dealt with in (7). When $|P|=2$, a basis for $W_{P}(N(P))$ is obtained from the indecomposable direct summands of $\left(k_{P}\right)^{N(P)}$. But these correspond, as in (2), to the principal representations of $k(N(P) / P)$, and it is readily seen that $W_{P}(N(P))$ is a homomorphic image of the projective ideal ${ }^{6}$ of $A(N(P) / P)$. Thus by $(7) W_{P}(N(P))$ is semisimple.

Now assume that $|P|=4$, and so $P \approx \mathscr{V}_{4}$. Write $H=N(P)$. Two cases arise:

(a) the centralizer $C(P)$ of $P$ in $H$ is $H$ itself, and

(b) the centralizer $C(P)$ is not $H$.

In case (a) it is clear that $H=R P$, the direct product of two groups. Thus by proposition 2

$$
A(H) \approx A(R) \otimes_{C} A(P) .
$$

Moreover in this correspondence

$$
W_{P}(H) \approx A(R) \otimes_{C} W_{P}(P) .
$$

$A(R)$ is semisimple and of finite dimension over $C$ as $(|R|, 2)=1$, and $W_{P}(P)$ is semisimple by $(13)$. Hence $W_{P}(H)$ is semisimple.

Case $(b)$. In this case we show $W_{P}(H)$ to be semisimple by taking an ideal $S$ of $W_{P}(H)$ such that both $S$ and $W_{P}(H) / S$ are semisimple. $W_{P}(H)$ is itself semisimple by proposition 1 .

Structure of $H$. We can find a complement $R$ to $P$ in $H$ and write $H=R P$. The centralizer $C(P)$ of $P$ in $H$ may be written in the form $Q P$, a direct product of groups, where $Q$ is a normal subgroup of $H$ contained in $R$. $H / Q P \approx R / Q$ has order 3 , as elements of $R / Q$ correspond to automorphisms of $\mathscr{V}$ whose orders are prime to 2. Take $r \in R$ such that $r Q$ generates $R / Q$. Then any element $h \in H$ has a unique expression in the form

$$
h=r^{\beta} q p \text {, }
$$

where $\beta=0,1,2, q \in Q, p \in P$. Write $\rho_{1}: H \rightarrow R$ to be the epimorphism $\rho_{1}(h)=r^{\beta} q$. We define $K$ to be the extension ${ }^{7}$ of $P$ by $R / Q$, its elements being written in the form $\left(r^{\beta} Q\right)(p)$ or $\left(r^{\beta}\right)(p)$ and satisfying the relation

$$
\left(r^{\beta}\right)(p)=\left(r^{\beta} p r^{-\beta}\right)\left(r^{\beta}\right)
$$

Thus $P$ is its own centralizer in $K$ and $K \approx A_{4}$. Further there is an epimorphism $\rho_{2}: H \rightarrow K$ given by $\rho_{2}(h)=\left(r^{\beta}\right)(p)$, where $h$ is given by (20). Finally we have a monomorphism,

$$
\rho: H \rightarrow R K,
$$

into the direct product of $R$ and $K$ given by $\rho(h)=\rho_{1}(h) \cdot \rho_{2}(h)$.

- In fact, $W_{-}(N(P)) \approx A_{0}(N(P) / P)$.

' $K \approx H / Q$ esentially. 
Indecomposable $k H$-modules. To obtain the indecomposable $k H$-modules, we use Higman's theorem 1 in [5] and look at the break-up of $k H$-modules $L^{H}$, where $L$ is an indecomposable $k Q P$-module. As in (3), $L$ has the form $M \# N$, where $M$ is an indecomposable (principal) $k Q$-module and $N$ is an indecomposable $k P$-module. By (4), $M \# N$ is stable in $H$ if and only if $M$ is stable in $R$ and $N$ is stable in $K$. By $(2)$ and $\left(2^{\prime}\right),(M \# N)^{H}$ is the direct sum of 3 non-isomorphic $k H$-modules

$$
(M \# N)^{\alpha} \text {, }
$$

if $M \# N$ is stable in $H$, or otherwise

$$
(M \# N)^{H}
$$

is indecomposable. In the latter case it should be noted that

$$
\left(r^{\beta} \otimes(M \# N)\right)^{H} \approx\left(\left(r^{\beta} \otimes M\right) \#\left(r^{\beta} \otimes N\right)\right)^{H} \approx(M \# N)^{H} .
$$

Moreover the vertex of an indecomposable $k H$-module so generated is the same as the vertex of $N$.

Now $W_{P}(H)=A_{P}(H) / A_{P}^{\prime}(H)=A(H) / A_{P}^{\prime}(H)$. Further $A_{P}^{\prime}(H) \geqq A_{\odot}(H)$, and so in looking at $W_{P}(H)$ we can work modulo the indecomposable $k H$ projectives. These last are in 1-1 correspondence with the indecomposable projectives of $k R$, for the regular $k P$-module $N$ is stable in $K$ as in (14i) and if $M$ is any indecomposable $k Q$-module, $M \# N$ is stable in $H$ if and only if $M$ is stable in $R$. Hence $(M \# N)^{H}$ decomposes just as $M^{R}$ does by (2).

Definition and semisimplicity of $S$. Consider the subspace $S$ of $W_{P}(H)$ spanned by classes of indecomposable $k H$-modules of the form $\left(M^{\prime} \# N^{\prime}\right)^{H}$ where $N^{\prime}$ is unstable in $K$. Then if $X$ is any $k H$-module such that $X_{(P Q)}$ has form $\oplus\left(M_{\alpha} \# N_{\alpha}\right)$, then

$$
\begin{aligned}
X \otimes\left(M^{\prime} \not N^{\prime}\right)^{H} & \approx \oplus\left(\left(M_{\alpha} \# N_{\alpha}\right) \otimes\left(M^{\prime} \# N^{\prime}\right)\right)^{H} \\
& \approx \oplus\left(\left(M_{\alpha} \otimes M^{\prime}\right) \not\left(N_{\alpha} \otimes N^{\prime}\right)\right)^{H} .
\end{aligned}
$$

The unstable classes $\{N\}$ span an ideal of $A(P)$ and so $S$ is an ideal of $W_{P}(H)$. Furthermore the map

$$
\left(M^{\prime} \not N^{\prime}\right)^{H} \rightarrow M^{\prime} \otimes_{C}\left(N^{\prime}\right)^{K}
$$

is an isomorphism from $S$ onto $A(Q) \otimes_{C} T$, where $T$ is the subspace of $A_{P}(K)$ coming from indecomposable $k P$-modules which are unstable in $K$. But from (18) $T$ is the direct sum of copies of $C$ and so $S$ is a semisimple ideal of $W_{P}(H)$.

$W_{P}(H) / S$. We now consider $W_{P}(H) / S$. Whereas the basis elements of $S$ came from $k P$-modules classes which were unstable in $K$, a basis of 
[10] The modular representation algebra of groups with Sylow 2-subgroup $Z_{2} \times Z_{2}$

$W_{P}(H) / S$ will be obtained from $k P$-module classes which have vertex $P$, and are stable in $K$.

The embedding homomorphism $\rho$ of $H$ into the direct product $R K$ as in (21) gives rise to an algebra homomorphism

$$
\rho^{*}: A(R K) \rightarrow A(H) \text {. }
$$

By proposition $2, A(R K) \approx A(R) \otimes_{C} A(K)$, and so we get a succession of homomorphisms:

$$
A(R) \otimes A(K) \approx A(R K) \rightarrow A(H) \rightarrow A(H) / A_{P}^{\prime}(H)=W_{P}(H) \rightarrow W_{P}(H) / S .
$$

Let $\sigma$ denote the composition of these homomorphisms. We show $\sigma$ is onto and analyse $W_{P}(H) / S$ as a quotient of $A(R) \otimes A(K)$.

$\sigma$ is onto. Let $N$ be an indecomposable $k P$-module which is stable in $K$, and let $\nu(p)(p \in P)$ be a representation afforded by this module. As $N$ is stable in $K$, there exists a matrix $R_{\nu}$ such that

Then

$$
\nu\left(r^{-1} p r\right)=R_{\nu}^{-1} v(p) R_{\nu} \quad(p \in P) .
$$

$$
v_{\alpha}(p)=v(p), \quad v_{\alpha}(r)=\omega^{\alpha} R_{\nu}, \quad(\alpha=0,1,2),
$$

are 3 inequivalent indecomposable $K$-representations "contained" in $N^{K}$.

Let $M$ be an indecomposable $k Q$-module. Let $\mu(q)(q \in Q)$ be a representation afforded by this module. Then the $k Q P$-module $M \# N$ is stable in $H$ if and only if $M$ is stable in $R$.

(i) Say $M$ is unstable in $R$. Let $\tilde{\mu}$ be the $R$-representation afforded by the indecomposable $k R$-module $M^{R}$. In the representation $\zeta$ afforded by $(M \# N)^{H}$ (indecomposable) choose a basis according to the direct sum decomposition

$$
\left((M \# N)^{H}\right)_{Q P}=\oplus\left(r^{\beta} \otimes M\right) \#\left(r^{\beta} \otimes N\right),
$$

but in the subspace corresponding to $r^{\beta} \otimes N$ choose the basis such that we have

$$
\zeta(q p)=\left[\begin{array}{ccc}
\mu(q) \otimes v(p) & 0 & 0 \\
0 & \mu\left(r^{-1} q r\right) \otimes v(p) & 0 \\
0 & 0 & \mu\left(r^{-2} q r^{2}\right) \otimes v(p)
\end{array}\right] .
$$

Then $\zeta(r)$ takes the form

$$
\zeta(r)=\left[\begin{array}{ccc}
0 & 0 & \mu\left(r^{3}\right) \otimes R_{\nu} \\
I \otimes R_{\nu} & 0 & 0 \\
0 & I \otimes R_{\nu} & 0
\end{array}\right]
$$

It is now clear that 


$$
\zeta(h)=\tilde{\mu}\left(\rho_{1}(h)\right) \otimes v_{0}\left(\rho_{2}(h)\right)
$$

for all $h \in H$. Thus $\left\{(M \# N)^{H}\right\}$ lies in the image of $\rho^{*}$.

(ii) Say $M$ is stable in $R$. Thus there exists a matrix $R_{\beta}$ such that

and

$$
\mu\left(r^{-1} q r\right)=R_{\mu}^{-1} \mu(q) R_{\mu},
$$

$$
\mu_{\alpha}(q)=\mu(q), \mu_{\alpha}(r)=\omega^{\alpha} R_{\mu}, \quad(\alpha=0,1,2),
$$

are 3 inequivalent indecomposable $R$-representations "contained" in $M^{R}$. Now

$$
(M \# N)^{H} \approx \stackrel{\oplus}{\oplus=0}_{\alpha=N}(M)^{\alpha},
$$

and we can take the representation $\zeta_{\alpha}$ afforded by $(M \not N)^{\alpha}$ to be in the form

$$
\zeta_{\alpha}(q p)=\mu(P) \otimes v(p), \zeta_{\alpha}(r)=\omega^{\alpha} R_{\mu} \otimes R_{\nu} .
$$

Thus again we have

$$
\zeta_{\alpha}(h)=\mu_{\alpha}\left(\rho_{1}(h)\right) \otimes \nu_{0}\left(\rho_{2}(h)\right) \quad(\alpha=0,1,2) .
$$

Hence $\sigma$ is onto $W_{P}(H) / S$.

Study of ker $\sigma$. Elements of the form

$$
\{L\} \otimes C_{n}^{*}(\mu) \quad\left(\mu \neq(\omega),\left(\omega^{2}\right)\right), \quad\{L\} \otimes D^{\alpha}
$$

of $A(R) \otimes A(K)$ ( $L$ an $k R$-module) either have vertex of order less than 4 or map to elements of $S$. Hence if $U$ is the ideal of $A(R) \otimes A(K)$ generated by the above elements, we can regard $\sigma$ as a map $\bar{\sigma}:(A(R) \otimes A(K)) / U \rightarrow$ $W_{P}(H) / S$. Moreover, from (18) the structure of $(A(R) \otimes A(K)) / U$ may be written

$$
(A(R) \otimes A(K)) / U \approx A(R) \otimes\left[\underset{\beta=0}{\oplus}\left(C\left[Y_{\beta}, \frac{1}{Y_{\beta}}\right]+\left\{\underset{\substack{n \geq 1 \\ \phi-U, \omega^{2}}}{\oplus} C I_{n \beta}(\phi)\right\rangle\right)\right] .
$$

This is semisimple, as $A(R)$ is the direct sum of a finite number of copies of $C$ and the direct factors on the right are each semisimple as is shown in $\S 4$ of [2].

$\bar{\sigma}$ and $\rho^{*}$. We next show that $(A(R) \otimes A(K)) / U$ is the ideal direct sum of three ideals, two of which are sent to 0 by $\bar{\sigma}$ and the last of which is isomorphic to $W_{P}(H) / S$ under $\bar{\sigma}$. To this end we look more closely at $\rho^{*}$.

If $M, N$ are indecomposable $k Q$-, $k P$-modules respectively with $N$ stable in $K$, then from (24i), (24ii) under $\rho^{*}$ we obtain

$$
M^{R} \otimes N^{\alpha} \rightarrow(M \# N)^{H} \text { when } M \text { is unstable in } R \text {, and }
$$

(26ii) $\quad M^{\beta} \otimes N^{\alpha} \rightarrow(M \# N)^{\alpha+\beta}$ when $M$ is stable in $R$ 
(superscripts being modulo 3). Clearly the only elements of $(A(R) \otimes A(K)) / U$ which can map onto these basis elements are in the subspace generated by $M^{R} \otimes N^{\alpha}$ or $M^{\beta} \otimes N^{\alpha}$ as the case may be.

(27) Thus in either case we have a subspace of dimension 3 mapping onto a 1-dimensional subspace (if we consider $\alpha+\beta$ fixed (modulo 3 ) in the second case).

Idempotents of $(A(R) \otimes A(K)) / U$. To obtain the ideal direct summands of $(A(R) \otimes A(K)) / U$ we proceed to obtain their generating idempotents as follows.

Let $E^{\alpha}, F^{\alpha}, G^{\alpha}(\alpha=0,1,2)$ be the 3 1-dimensional $k R-, k K-, k H-$ modules respectively corresponding to the matrix representations

$$
r^{\beta} \rightarrow \omega^{\beta \alpha}
$$

Thus we can write $k_{R}=E^{0}, k_{K}=F^{0}, k_{H}=G^{0}$. We use the same symbols $E^{\alpha}, F^{\alpha}, G^{\alpha}$ to denote the corresponding module classes. Then under $\rho^{*}$ we have from (26ii) that

$$
E^{\alpha} \otimes F^{\beta} \rightarrow G^{\alpha+\beta}
$$

Consider the normal subgroup $Q P$ of $R K$. $R K / Q P \approx R / Q \cdot K / P$, which is the direct product of two cyclic groups of order 3 . We can denote the various $k(R / Q \cdot K / P)$-module classes by $E^{\alpha} \otimes_{C} F^{\beta}(\alpha, \beta=0,1,2)$. Thus we get that $A(R / Q \cdot K / P)$ is the direct sum of 9 copies of $C$ with idempotents

$$
I_{\alpha \beta}=\frac{1}{3}\left(E^{0}+u^{\alpha} E^{1}+u^{2 \alpha} E^{2}\right) \otimes \frac{1}{3}\left(F^{0}+u^{\beta} F^{1}+u^{2 \beta} F^{2}\right) .
$$

where $\alpha, \beta=0,1,2$, and $u$ is a primitive cube root of unity in $C$. By proposition 4 we get a corresponding decomposition of $A(R K) \approx A(R) \otimes A(K)$, and so one induced on the quotient $(A(R) \otimes A(K)) / U$. Consider the 3 idempotents

$$
\begin{aligned}
& J_{0}=I_{00}+I_{11}+I_{22}=\frac{1}{3}\left(E^{0} \otimes F^{0}+E^{1} \otimes F^{2}+E^{2} \otimes F^{1}\right), \\
& J_{1}=I_{10}+I_{21}+I_{02}=\frac{1}{3}\left(E^{0} \otimes F^{0}+u E^{1} \otimes F^{2}+u^{2} E^{2} \otimes F^{1}\right), \\
& J_{2}=I_{20}+I_{01}+I_{12}=\frac{1}{3}\left(E^{0} \otimes F^{0}+u^{2} E^{1} \otimes F^{2}+u E^{2} \otimes F^{1}\right) .
\end{aligned}
$$

Then $\rho^{*}\left(J_{0}\right)=G^{0}$, the identity of $A(H)$, and $\rho^{*}\left(J_{1}\right)=\rho^{*}\left(J_{2}\right)=0$. On the other hand none of the following products vanishes:

$$
\begin{array}{ll}
J_{\beta} \cdot\left(M^{R} \otimes N^{\alpha}\right) & (M \text { unstable in } R), \\
J_{\beta} \cdot\left(M^{\beta} \otimes N^{\alpha}\right) & (M \text { stable in } R),
\end{array}
$$

where $\beta=0,1,2$, and the 3-dimensional subspaces of (27) are the sum of 1 -dimensional subspaces one in each of the summands $J_{\beta} \cdot A(R K)(\beta=0,1,2)$. 
Hence restricting $\bar{\sigma}$ to the direct summand $J_{0} \cdot(A(R) \otimes A(K)) / U$ we have that $\bar{\sigma}$ is one-to-one and onto. Thus

$$
W_{P}(H) / S \approx J_{0} \cdot(A(R) \otimes A(K)) / U
$$

Hence $W_{P}(H) / S$ is isomorphic to an ideal (direct summand) of a semisimple algebra (by (25)) and so $W_{P}(H) / S$ is semisimple.

$W_{P}(H)$ contains an ideal $S$ such that $W_{P}(H) / S$ and $S$ are semisimple and so by proposition 1 it is semisimple. This completes the proof of the semisimplicity of $W_{P}(N(P))$ for $P$ of orders 1, 2 or 4. By Green's theorem $(6), A(G)$ is semisimple. By (8) we can further remove the restriction of $k$ being algebraically closed and so we have the following theorem:

THEOREM. Let $G$ be a finite group whose Sylow 2-subgroup is isomorphic to $Z_{2} \times Z_{2}$, and let $k$ be any field of characteristic 2 . Then the modular representation algebra $A(G)$ formed from $k G$-modules is semisimple.

\section{References}

[1] Conlon, S. B., Twisted group algebras and their representations, J. Austral. Math. Soc. 4 (1964), $152-173$.

[2] Conlon, S. B., Certain representation algebras, J. Austral. Math. Soc. 5 (1965), 83-99.

[3] Curtis, C. W. and Reiner, I., Representation theory of finite groups and associative algebras, Interscience, New York, 1962.

[4] Green, J. A., A transfer theorem for modular representations, J. of Algebra 1 (1864), $73-84$.

[5] Higman, D. G., Indecomposable representations at characteristic $p$, Duke Math. J. 21 (1964), $377-381$.

University of Sydney 